\title{
Considering respiratory tract infections and antimicrobial sensitivity: An exploratory analysis
}

\author{
Amin, R. ${ }^{1}$, Hoque A. M. W. ${ }^{2}$, Khan R. F. ${ }^{3}$ and Rahman M. ${ }^{*}$ \\ ${ }^{1}$ Department of Microbiology and Immunology, Bangabandhu Sheikh Mujib Medical University (BSMMU), Shahbag, \\ Dhaka 1000, Bangladesh. \\ ${ }^{2}$ ICDDR,B , Mohakhali, Dhaka 1213, Bangladesh. \\ ${ }^{3}$ Medical Affairs Division, Sanofi-aventis Bangladesh Limited, 6/2/A Segun Bagicha, Dhaka 1000, Bangladesh. \\ ${ }^{4}$ Department of Epidemiology, Mailman School of Public Health, Columbia University, NY, USA. \\ E-mail:mr2640@columbia.edu
}

Received 7 March 2009; received in revised form 1 June 2009; accepted 2 July 2009

\begin{abstract}
This study was conducted to observe the sensitivity and resistance of status of antibiotics for respiratory tract infection $(\mathrm{RTI})$. Throat swab culture and sensitivity report of 383 patients revealed sensitivity profiles were observed with amoxycillin (7.9\%), penicillin (33.7\%), ampicillin (36.6\%), co-trimoxazole (46.5\%), azithromycin (53.5\%), erythromycin $(57.4 \%)$, cephalexin $(69.3 \%)$, gentamycin $(78.2 \%)$, ciprofloxacin $(80.2 \%)$, cephradine $(81.2 \%)$, ceftazidime $(93.1 \%)$, ceftriaxone (93.1\%). Sensitivity to cefuroxime was reported $93.1 \%$ cases. Resistance was found with amoxycillin $(90.1 \%)$, ampicillin $(64.1 \%)$, penicillin (61.4\%), co-trimoxazole (43.6\%), erythromycin (39.6\%), and azithromycin (34.7\%). Cefuroxime demonstrates high level of sensitivity than other antibiotics and supports its consideration with patients with upper RTI.
\end{abstract}

Keywords: sensitivity, Respiratory tract infection (RTI)

\section{INTRODUCTION}

Respiratory tract infection (RTI) is considered as one of the major public health problems in developing countries. It occurs both among children and adults. It is recognized as the leading cause of morbidity and mortality in many developing countries (Baqui et al., 2007). In developing countries thirty percent of all patients' consultation and twenty five percent of all pediatric admission are of acute respiratory tract infections (Rahman and Rahman, 1997) and which ultimately causes 3.5 million deaths in children each year (Hart and Kariuki, 1998). However, most infections are limited to the upper respiratory tract and only $5 \%$ involve the lower respiratory tract, respectively. Recent studies showed that Streptococcus pneumonie, Haemophilus influenzae and respiratory syncytial virus are the main causes of RTI in children (Rudan et al., 2008). With effective management and appropriate drug RTI can be managed successfully (Chowdhury et al.,2008).

Antibiotics are commonly prescribed in treating common respiratory tract infections even with viral etiology. As there is difficulty in establishing bacterial etiology at the time of prescription, antibacterial therapy of RTIs is usually empirical considering the presence of risk factors and severity of disease. In doing so, this misuse of antibiotics has led to the development of antibioticresistant bacteria. Nowadays, antimicrobial resistance is a recognized problem throughout the world (Finch, 2004). Excessive use of antibiotics in the outpatient setting has contributed to the increase in antimicrobial resistance (Dowell and Schwartz, 1997; Diekema et al., 2000; Brown et al., 2003). Resistance of antimicrobials in respiratory tract infection is also a common health problem both for adult and children in Bangladesh. As in other developing countries, resistance to commonly used antibiotics is also increasing in Bangladesh due to its indiscriminate use and prescription. Clinical studies have shown that many penicillin-resistant pneumococci are also resistant to chloramphenicol, and cephalosporins such as cefuroxime and ceftriaxone, thus limiting treatment options (Sharma et al., 2005). The emergence of antibiotic resistance in common respiratory pathogens may jeopardize the effectiveness of empiric treatment causing increased morbidity and mortality from the diseases and also increased health cost to the patient. Accurate bacteriologic records of throat swab culture results may provide guidance for empiric therapy before sensitivity patterns are available.

Therefore, knowledge of the local prevalence of pathogens and their antimicrobial sensitivity patterns is essential for clinicians in their routine work. Clinicians should also be aware of the sensitivity patterns in both neighbouring and distant areas. There is a lack of data in Bangladesh on commonly used antimicrobials in respiratory tract infections including cefuroxime sensitivity and/or resistance on respiratory tract pathogens. Therefore our study aims to determine the magnitude of existing problem of antibiotic resistance by observing 
commonly used antimicrobial especially cefuroxime sensitivity and resistance status in respiratory tract pathogens.

\section{MATERIALS AND METHODS}

This cross-sectional survey was conducted between May to July 2007 to identify commonly used antimicrobials especially cefuroxime sensitivity and/or resistance in respiratory tract pathogens in Bangladesh. Clinically suspected outpatients of upper respiratory tract infections were observed for culture and sensitivity test of throat swabs. Throat swabs were collected from 383 cases from four major diagnostic centres in Dhaka city. The collected samples were stratified according to the growth of any pathogenic bacteria to evaluate sensitivity and/or resistance of commonly used antimicrobials. Antimicrobial susceptibility tests were performed on the isolates using disk diffusion methods. Proportion of commonly used antimicrobials including cefuroxime sensitivity and resistance was determined.

Statistical power and sample size justification: Since there is no previous data on prevalence, we have assumed $\rho=0.50$; Type I error of 0.05 . Finally the sample size was 383. This sample size allowed having an optimum number of patients in each centre.

\section{RESULTS}

As shown in Table 1, a total of 384 clinically suspected cases of upper respiratory tract infections, among them only 383 throat swab samples were considered for final analysis. Mean age of the patients was 23 years and half of them were children and others were teenagers and adults. Among all the samples, one among every four cases showed growth of any pathogenic bacteria in culture and sensitivity test.

Table 1: Distribution of bacterial growth among all participants. Cross sectional study, Dhaka, Bangladesh

\begin{tabular}{lcc}
\hline Bacterial growth & N & Percentage (\%) \\
\hline Growth & 101 & 26.3 \\
No growth & 282 & 73.7 \\
Total & 383 & 100.0 \\
\hline
\end{tabular}

According to the report of culture and sensitivity test, the isolated pathogens in the aggregates were $\beta$ hemolytic streptococci $(28.7 \%)$, Klebsiella pneumoniae $(28.7 \%)$, Staphylococci (25.7\%), Pseudomonas (4\%), E. coli (2\%), Pneumococcus (2\%) (Table 2).

Among the commonly used antimicrobials in URTI, sensitivity profiles were observed with amoxycillin (7.9\%), penicillin (33.7\%), ampicillin (36.6\%), co-trimoxazole (46.5\%), azithromycin (53.5\%), erythromycin (57.4\%), cephalexin $(69.3 \%)$, gentamycin $(78.2 \%)$, ciprofloxacin (80.2\%), cephradine (81.2\%), levofloxacin (86.2\%), ceftazidime $(93.1 \%)$, ceftriaxone $(93.1 \%)$. Sensitivity to cefuroxime was reported in $93.1 \%$ cases. However, antimicrobial resistance was observed most against amoxycillin $(90.1 \%)$. Resistance to others was as follows, penicillin (61.4\%), ampicillin (64.1\%), co-trimoxazole (43.6\%), erythromycin (39.6\%), and azithromycin (34.7\%) (Table 3).

Table 2: Bacterial isolates in culture

\begin{tabular}{lcc}
\hline \multicolumn{1}{c}{ Isolated pathogens } & N & Percentage (\%) \\
\hline B hemolytic streptococci & 29 & 28.7 \\
Klebsiella pneumoniae & 29 & 28.7 \\
Pseudomonas & 4 & 4.0 \\
Staphylococci & 26 & 25.7 \\
Pneumococcus & 2 & 2.0 \\
E. coli & 2 & 2.0 \\
Total & 92 & 91.1 \\
Misssing & 9 & 8.9 \\
Total & 101 & 100.0 \\
\hline
\end{tabular}

Table 3: Commonly used antimicrobial sensitivity and resistance status in URTI. Cross sectional study, Dhaka, Bangladesh

\begin{tabular}{lcc}
\hline \multicolumn{1}{c}{ Antibiotics } & Sensitivity (\%) & Resistance (\%) \\
\hline Amoxycillin & 7.9 & 90.1 \\
Penicillin & 33.7 & 64.4 \\
Ampicillin & 36.6 & 61.4 \\
Co-trimoxazole & 46.5 & 43.6 \\
Azithromycin & 53.5 & 34.7 \\
Erythromycin & 57.4 & 39.6 \\
Cephalexin & 69.3 & 26.7 \\
Gentamycin & 78.2 & 19.8 \\
Ciprofloxacin & 80.2 & 9.9 \\
Cephradine & 81.2 & 15.8 \\
Levofloxacin & 86.2 & 11.9 \\
Ceftazidime & 93.1 & 4.0 \\
Ceftriaxone & 93.1 & 4.0 \\
Cefuroxime & 93.1 & 6.9 \\
\hline
\end{tabular}

Among 383 reports, 101 (26.3\%) were growth positive, while $282(73.4 \%)$ did not show any growth of pathogenic bacteria. Of the respondents, about $93.1 \%$ cases reported sensitivity to cefuroxime (Figure 1).

\section{DISCUSSION}

Antimicrobial drugs are an important resource that must be conserved for proper use. To accomplish this requires knowledge of the dimension of the problem and early warnings of the emergence of resistant isolates. Antibiotic resistant bacteria are associated with the failure of treatment and increased mortality and morbidity. Many bacteria (for example, $\beta$ hemolytic streptococci, $K$. pneumoniae, Staphylococci) still remaining predictably 


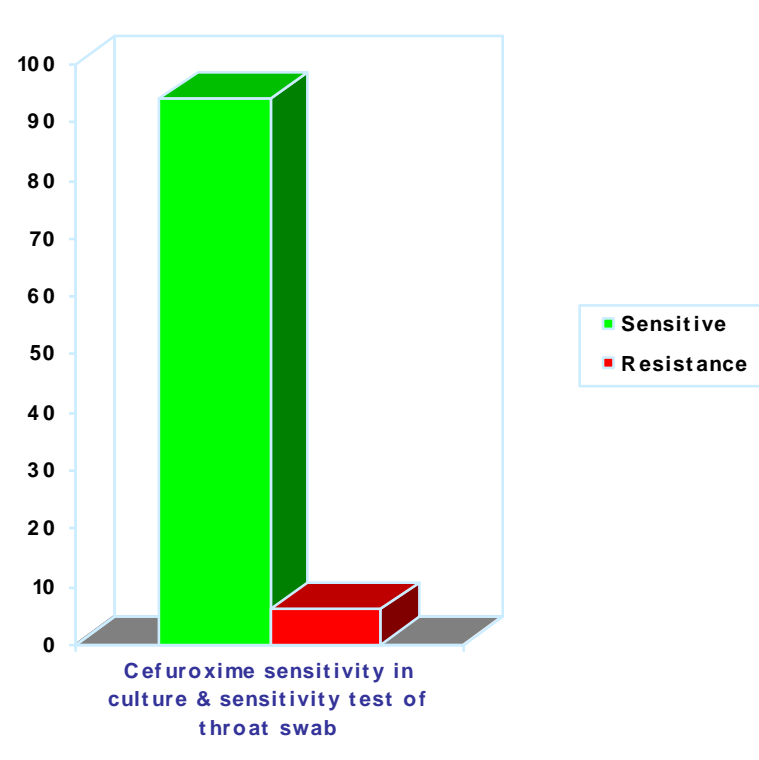

Figure 1: Cefuroxime sensitivity in culture \& sensitivity test of throat swabs

sensitive to a few of the routinely available antimicrobial drugs. This study result demonstrates that most of the bacterial isolates in culture and sensitivity of throat swabs are sensitive to cefuroxime (93.1\%). In a study at Peshwar Pakistan found, amoxicillin as the best cost effective drug followed by clarithromycin and cefuroximine in the treatment of RTI (Aurangzeb and Hameed, 2003). A study was carried out in the largest children hospital in Bangladesh concluded that trimethoprim suphamethoxazole resistance is very common in treatment of RTI for children under the age of five (Baqui et al., 2007). However, sensitivity to penicillin, ampicillin, amoxycillin, co-trimoxazole, erythromycin, azithromycin has continued to fall and precludes their use in empiric treatment. Among them, a high level of resistance was observed to amoxycillin (90.1\%). Perhaps this occurred, as the study centres were in urban setting and the patients have more access to the doctors in comparison to others parts in the country.

The increasing resistance of these antimicrobials in upper respiratory tract pathogens is of great concern; our study had a high level of resistance to some of these commonly used antimicrobial drugs. The high prevalence of resistant bacteria seems to be related to irrational antimicrobial usage. A meta analysis concluded, oral cephalosporin is significantly more effective than oral pencillin in the treatment of RTI (Casey and Pichichero, 2004). In Bangladesh the use of antimicrobial drugs in URTI is uncontrolled; antibiotics can be purchased in pharmacies, general stores, and even market stalls. Many of the medical practitioners prescribe antibiotics to these patients on the basis of symptoms alone. Selection of an anti-infective agent regimen for the treatment of upper respiratory tract pathogens should be based on the drug's spectrum of activity as well as the regimen's bacteriologic and clinical efficacy, potential adverse effects, ease of administration and patient compliance, and cost. Patients sometimes expect an antibiotic for an acute respiratory infection; health care professionals strive for patient satisfaction to some extend. Patients sometimes insist physicians to prescribe antibiotics without any specific reason. In studies carried out in Bangladesh, it was observed in many cases that physicians are requested either by patients or their attendants to prescribe antibiotic as they perceive that it will enhance quick recovery. If the diagnosis is a viral illness, the physician needs to have a contingency plan to explain to the patient why an antibiotic will not be prescribed. The different international authorities have endorsed a campaign to promote appropriate antibiotic use for the treatment of acute respiratory tract infections in adults and children. It is also documented that Clinical Practice Guidelines providing evidence-based recommendations to be practiced by the physicians for the proper management of patients (Gonzales et al., 2001). These provide practical strategies for limiting antibiotic use to the patients with URTI. Furthermore, it is required to restrict the sale of antibiotics without prescription, to use antibiotics more judiciously in hospitals by intensive teaching of the principles of the use of antibiotics (Kim and Park, 1998). There must also be greater regulation of the use of antibiotics; this should be accompanied by strategies to educate the public and doctors on the appropriate use of antibiotics in URTI (Hart and Kariuki, 1998).

The survey shows an increased level of resistance among the respiratory pathogens against commonly used antimicrobials in upper respiratory tract infections in Bangladesh. On the other hand few of the antimicrobial drugs still remain highly sensitive to the pathogens. Among them cefuroxime, ceftazidime and ceftriaxone demonstrate high level of sensitivity on respiratory tract pathogens. This study supports use of cefuroxime as appropriate in the patients with URTI. Morover cefuroxime is found safe during pregnancy (Benyamini et al., 2005).

\section{ACKNOWLEDGEMENT}

This study was funded by an unrestricted educational grant of sanofi-aventis Bangladesh Limited. We would like to express thanks to all the laboratories for providing the data. We express special thanks to the study investigators: Prof Samir K Saha, Prof Kaniz Moula, Dr. Ferdous Ahmed, Dr Lutfun Nessa and Dr Javed Sobhan for their hard work and commitment.

\section{REFERENCES}

Aurangzeb, B. and Hameed, A. (2003). Comparative efficacy of amoxicillin, cefuroxime and clarithromycin in the treatment of community-acquired pneumonia in children. Journal of College of Physicians and Surgeons Pakistan 13, 704-707.

Baqui, A. H., Rahman, M., Zaman, K., El Arifeen, S., Chowdhury, H. R., Begum, N., Bhattacharya, G., 
Chotani, R. A., Yunus, M., Santoshams, M. and Black, R. E. (2007). A population-based study of hospital admission incidence rate and bacterial aetiology of acute lower respiratory infections in children aged less than five years in Bangladesh. Journal of Health, Population and Nutrition 25, 179188.

Benyamini, L., Merlob, P., Stahl, B., Braunstein, R., Bortnik, O., Bulkowstein, M., Zimmerman, D. and Berkovitch, M. (2005). The safety of amoxicillin/clavulanic acid and cefuroxime during lactation. Therapeutic Drug Monitoring 27, 499-502.

Brown, D. W., Taylor, R., Rogers, A., Weiser, R. and Kelley, M. (2003). Antibiotic prescriptions associated with outpatient visits for acute upper respiratory tract infections among adult Medicaid recipients in North Carolina. North Carolina Medical Journal 64, 148156.

Casey, J. R. and Pichichero, M. E. (2004). Meta-analysis of cephalosporin versus penicillin treatment of group A streptococcal tonsillopharyngitis in children. Pediatrics 113, 866-882.

Chowdhury, E. K., El Arifeen, S., Rahman, M., Hoque, D. E., Hossain, M. A., Begum, K., Siddik, A., Begum, N., Sadeq-ur Rahman, Q., Akter, T., Haque, T. M., Al-Helal, Z. M., Baqui, A. H., Bryce, J. and Black, R. E. (2008). Care at first-level facilities for children with severe pneumonia in Bangladesh: a cohort study. Lancet 372, 822-830.

Diekema, D. J., Brueggemann, A. B. and Doern, G. V. (2000). Antimicrobial-drug use and changes in resistance in Streptococcus pneumoniae. Emerging Infectious Diseases 6, 552-556.

Dowell, S. F. and Schwartz, B. (1997). Resistant pneumococci: protecting patients through judicious use of antibiotics. American Family Physician 55, 1647-1654 and 1657-1658.

Finch, R.G. (2004). Antibiotic resistance: a view from the prescriber. Nature Reviews Microbiology 2, 989-994.

Gonzales, R., Bartlett, J. G., Besser, R. E., Cooper, R. J., Hickner, J. M., Hoffman, J. R. and Sande, M. A. (2001). Principles of appropriate antibiotic use for treatment of acute respiratory tract infections in adults: background, specific aims, and methods. Annals of Internal Medicine 134, 479-486.

Hart, C. A. and Kariuki, S. (1998). Antimicrobial resistance in developing countries. British Medical Journal 317, 647-650.

Kim, W. J. and Park, S. C. (1998). Bacterial resistance to antimicrobial agents: an overview from Korea. Yonsei Medical Journal 39, 488-494.

Rahman, M. M. and Rahman, A. M. (1997). Prevalence of acute respiratory tract infection and its risk factors in under five children. Bangladesh Medical Research Council Bulletin 23, 47-50.

Rudan, I., Boschi-Pinto, C., Biloglav, Z., Mulholland, K. and Campbell, H. (2008). Epidemiology and etiology of childhood pneumonia. Bulletin of the World Health Organization 86, 408-416.
Sharma, R., Sharma, C. L. and Kapoor, B. (2005). Antibacterial resistance: current problems and possible solutions. Indian Journal of Medical Sciences 59, 120-129. 